\title{
Electric Double-Layer Capacitor Fabricated with Addition of Carbon Nanotube to Polarizable Electrode
}

\author{
Yoshiyuki Show \\ Department of Electrical and Electronic Engineering, Tokai University, 4-1-1 Kitakaname, Hiratsuka, Kanagawa, 259-1292, Japan \\ Correspondence should be addressed to Yoshiyuki Show, show@tokai-u.jp \\ Received 13 October 2011; Accepted 18 March 2012 \\ Academic Editor: Sulin Zhang \\ Copyright () 2012 Yoshiyuki Show. This is an open access article distributed under the Creative Commons Attribution License, \\ which permits unrestricted use, distribution, and reproduction in any medium, provided the original work is properly cited.
}

Electrical double-layer capacitor (EDLC) was fabricated with addition of carbon nanotube (CNT) to polarization electrodes as a conducting material. The CNT addition reduced the series resistance of the EDLC by one-twentieth, while the capacitance was not increased by the CNT addition. The low series resistance leaded to the high electrical energy stored in the EDLC. In this paper, the dependence of the series resistance, the specific capacitance, the energy, and the energy efficiencies on the CNT addition is discussed.

\section{Introduction}

Electric double-layer capacitors (EDLCs), supercapacitors, or ultracapacitors have the same type of electrical energy storage device, which is based on a double electric charge layer effect $[1,2]$. Commercial EDLCs recently have capacitance value up to thousands $\mathrm{F}$ and the power density up to thousands $\mathrm{W} / \mathrm{kg}$, which is higher than that of conventional batteries $(<1000 \mathrm{~W} / \mathrm{kg})$. Higher capacitance and power density allow higher energy to be stored and discharged at higher rate. Moreover, EDLCs have longer cycle lives exceeding 500000 times. These features have generated great interest in the application of EDLCs such as consumer electronics, hybrid electric vehicle [3], and industrial power managements. On the other hand, the energy density of EDLCs is below $10 \mathrm{Wh} / \mathrm{kg}$, which is lower than that of batteries (35-40 Wh/kg of lead-acid batteries, $-150 \mathrm{Wh} / \mathrm{kg}$ of lithium ion batteries). The electrical energy ( $\left.L_{\text {stored }}\right)$ stored in the EDLC is calculated according to the following equation:

$$
L_{\text {stored }}=\frac{1}{2} C V^{2} \text {, }
$$

where $C$ is capacitance and $V$ is voltage. This equation indicates that an increase in the capacitance leads the electrical energy stored in the EDLC to be high.
The capacitance of the EDLC is theoretically proportional to surface area $(S)$ of polarizable electrodes, as based on the following equation:

$$
C=\int \frac{\varepsilon_{0} \varepsilon_{r}}{d} S
$$

where $\varepsilon_{0}$ and $\varepsilon_{r}$ are permittivity in vacuum and relative permittivity of the double layer, respectively, and $d$ is thickness of double layer. Therefore, activated carbon is widely used as a constituent of polarizable electrode in order to obtain large capacitance, because it has high surface area of over $1000 \mathrm{~m}^{2} / \mathrm{g}$.

In addition to the surface area of the polarizable electrodes, the energy density is also gravened by the series resistance of the EDLC, because the charge and the discharge currents are followed in the series resistance. The energy loss $\left(L_{\text {loss }}\right)$, which is caused by the current flowing in the serial resistance during the charge or the discharge, is estimated using the following equation:

$$
L_{\mathrm{loss}}=I^{2} R_{s} \cdot t,
$$

where $I$ is the charge or the discharge current, $R_{s}$ is the series resistance, and $t$ is the charge or the discharge time. In the application of the EDLC to the second power system in an electric vehicle, the discharge for the acceleration and the charge by the deceleration take place over a short 
period. Therefore, these charge and discharge currents are of a particularly high value when compared with those of other applications. Higher charge and discharge currents cause the energy loss to be increased. For these reasons, there is a strong requirement for the series resistance to be decreased in order to reduce the energy loss.

A decrease in series resistance $\left(R_{s}\right)$ also allows the power density of EDLC to be increased, because maximum output power $\left(P_{\max }\right)$ of EDLCs is theoretically calculated to the following equation:

$$
P_{\max }=\frac{V^{2}}{4 R_{s}},
$$

where $V$ is the voltage. Therefore, the series resistance of EDLCs is also required to be reduced in order to increase the energy and the power densities.

Activated carbon is the base material widely used for the fabrication of polarizable electrodes $[4,5]$. However, the activated carbon in itself does not have a high enough electrical conductance to act as a fully suitable material for the polarizable electrodes. An EDLC fabricated exclusively from activated carbon shows a high energy loss during the charge and the discharge processes, because it has high series resistance. Therefore, a conducting material such as acetylene black is generally added to the polarizable electrodes in order to decrease the series resistance.

Carbon nanotube (CNT) is one of the candidate materials for polarizable electrodes of the EDLC. The CNT is tube shaped with a diameter of a few nm, which is expected to be suitable for forming the double layer between the surface and the electrolyte. Moreover, CNTs have a high electrical conductivity [6] and chemical stability. It has been reported that the EDLC fabricated from multiwalled CNT showed high specific capacitance in high range of 4-135 F/g $[7,8]$. For single-walled CNT, the specific capacitance of $180 \mathrm{~F} / \mathrm{g}$ and the power density of $20 \mathrm{~kW} / \mathrm{kg}$ at the energy density of $7 \mathrm{Wh} / \mathrm{kg}$ were reported [9]. Moreover, CNTbased electrodes fabricated by direct synthesis of CNT on the bulk $\mathrm{Ni}$ substrates have been reported to have the specific capacitance of $38.7 \mathrm{~F} / \mathrm{kg}$ [10]. However, a comparative investigation of the specific capacitance, achieved with CNTs and activated carbon materials, revealed that activated carbon materials exhibited significantly higher capacitance [11]. On the other hand, the addition of CNT to activated carbon instead of acetylene black or graphite power for the polarizable electrodes also has been studied [12-15].

In this paper, CNTs are added to the polarizable electrode which is made of activated carbon. The CNT concentration in the polarizable electrode is from 0 to $100 \%$. The dependence of the series resistance, the specific capacitance, the electrical energy, and the energy efficiency on the CNT addition is studied.

\section{Experimental Conditions}

CNT (Ohashi Kasga Tsusho Inc.) and activated carbon (Hohsen Corp.) were used as the base material for the polarizable electrode. The ratio of the CNT to the activated carbon was varied from 0 (pure activated carbon) to $100 \%$ (pure CNT). These electrode materials were mixed in a mortar made of agate. Polytetrafluoroethylene (PTFE) was added to the mixture of CNT and activated carbon as binder at $17 \%$ in weight. Acetylene black (Kanto Chemical Co., Inc) was also mixed to some of the polarizable electrodes instead of the CNTs for reference/comparison purposes. The total mass of the polarizable electrode was varied from $30 \mathrm{mg}$ to $120 \mathrm{mg}$.

The mixture was pressed at $20 \mathrm{MPa}$ and formed into a disk shape with an area of $1 \mathrm{~cm}^{2}$ (diameter of $11.5 \mathrm{~mm}$ ) using a steel mold-type pressure apparatus. The thickness of the polarizable electrode hardly depends on the ratio of the constituents. In this experiment, it was varied form 0.5 to $2 \mathrm{~mm}$ by increasing its weight from 30 to $120 \mathrm{mg}$.

The constituents of the polarizable electrode were characterized by the scanning electron microscope (SEM) (Hitachi High-Technologies Corp., HF-2200TU and S-4800). The capacitance measurements were performed using a symmetrical cell configuration (a two-electrode system). The platinum plates were used as a collector electrode, because contact resistance between the polarizable electrode and the platinum plate is lower than any other metal such as stainless steel. A pair of polarizable electrodes was set up in a glass beaker or a can cell to provide both an anode and a cathode. An organic solvent (1.5 M SBP-BF 4 /PC, Japan Carlit Co., Ltd.) was used as the electrolyte. This electrolyte has high electrical conductivity of $16 \mathrm{mS} / \mathrm{cm}$.

The constant current charge-discharge measurement was carried out at $5 \mathrm{~mA}$ in the voltage range between 0.1 and 1.2 V using the source meter (Advantest Corp., R6243). The voltage change during the charge-discharge was measured. The series resistance and the capacitance of the EDLC were estimated from the voltage change by the mathematical fitting method described in Result and Discussion.

\section{Result and Discussion}

Figure 1 shows SEM images of (a) activated carbon (AC), (c) carbon nanotube (CNT), and (e) acetylene black (AB), which are constituents of the polarizable electrodes. The activated carbon was a particle with a size of $2-3 \mu \mathrm{m}$. A high-resolution SEM image shows that the activated carbon contains a great number of mesopores on its surface. The nitrogen adsorption measurements indicated that the activated carbon was $2000 \mathrm{~m}^{2} / \mathrm{g}$ in the specific surface area. The CNT was cylindrical-shaped material as shown in Figure 1(c). The diameter and the length were approximately $10 \mathrm{~nm}$ and $100 \mu \mathrm{m}$, respectively. No traces of the metal catalyst, which had been used for CNT growth, were found at the tips of the CNT in this SEM image. Furthermore, very little amorphous carbon, which is a byproduct of the CVD process, was found. This SEM investigation suggests that the CNT used is well purified by the post-acid washing process used after the CNT growth. The acetylene black used was a particle with a diameter of $50-60 \mathrm{~nm}$, as shown in Figure 1(e). 


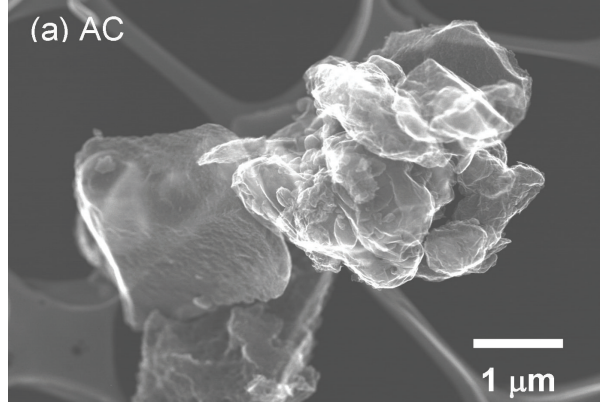

(a)

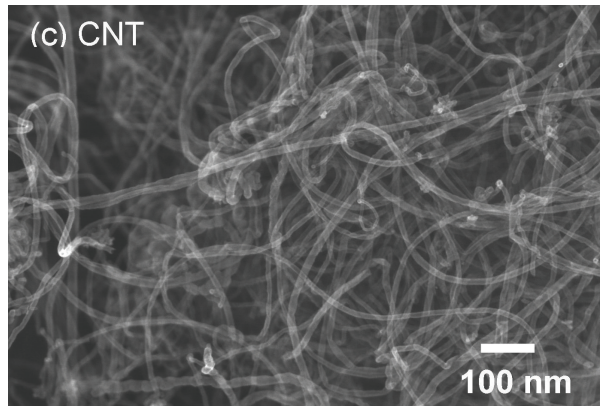

(c)

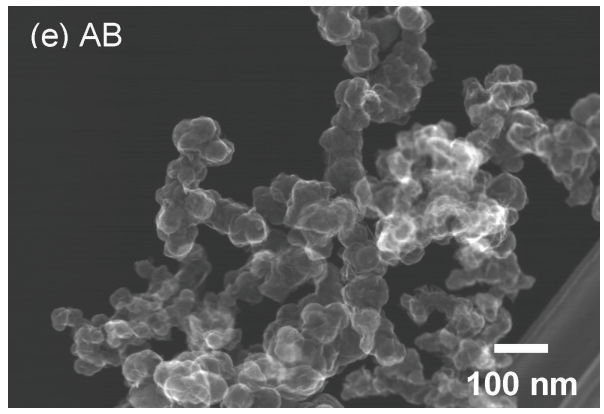

(e)

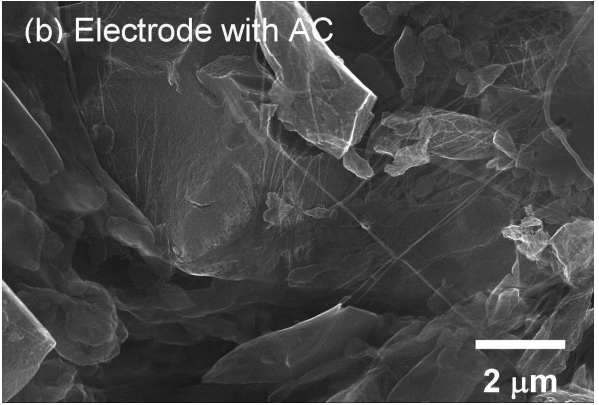

(b)

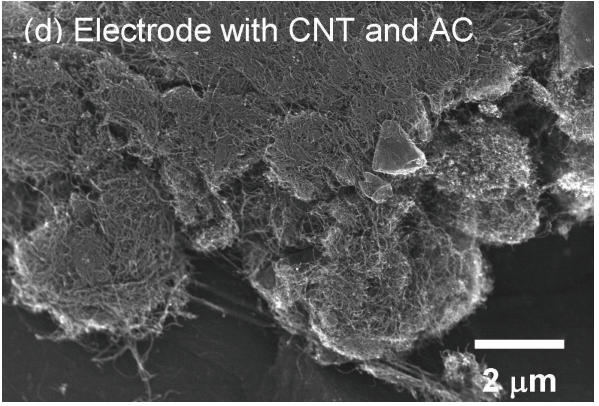

(d)

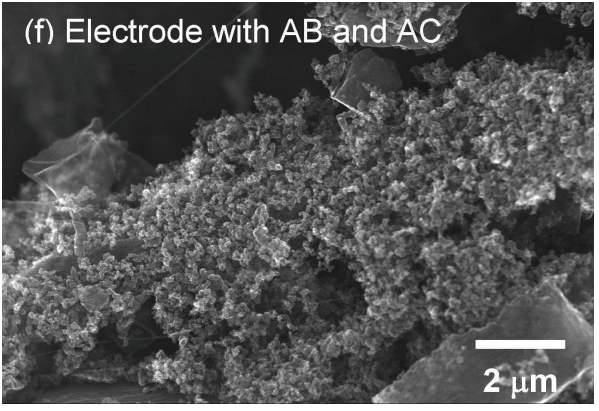

(f)

Figure 1: SEM images of (a) activated carbon (AB), (c) carbon nanotube (CNT), and (e) acetylene black (AB) as constituents of the polarizable electrodes. SEM images of polarizable electrodes fabricated from (b) exclusively activated carbon, (d) CNT and activated carbon, and (f) acetylene black and activated carbon.

Figure 1 also shows SEM images of polarizable electrodes fabricated from (b) exclusively activated carbon, (d) CNT and activated carbon, and (f) acetylene black and activated carbon. The concentration of CNT or acetylene black in polarizable electrode is $20 \%$. Particles of activated carbon are stacked each other by the polytetrafluoroethylene (PTFE), which was added as binder as shown in Figure 1(b). Thin fiber-shaped PTFE was observed in this image. On the other hand, the activated carbon particles were covered with the CNT in the polarizable electrode containing CNT, as shown in Figure 1(d). The CNTs kept their long shape and were intertwisted on many particles of the activated carbon in the polarizable electrode. The SEM image of the acetylene black in Figure 1(f) shows their particles are distributed on the activated carbon surface.

Figure 2 shows the charge-discharge curves of EDLCs fabricated with the following three types of polarizable electrodes: (a) those of exclusively activated carbon (no
CNT) and those containing (b) 10 and (c) 50\% CNTs. Those polarizable electrodes were fixed at the weight of $60 \mathrm{mg}$. The charge-discharge measurements were carried out at a constant current of $5 \mathrm{~mA}$. A rapid voltage change at the initial stage of the charge and the discharge, which is called the IR drop, was observed for all types of EDLCs. The IR drops of $0.45 \mathrm{~V}$ and $0.04 \mathrm{~V}$ were observed for (a) the EDLC without and (b) the EDLC with the 10\% CNT addition, respectively. At $0.04 \mathrm{~V}$ the IR drop of the EDLC containing CNT at $10 \%$ was lower than that of the EDLC fabricated without using CNT.

The voltage gradually increased with an increase in the charging time after the initial stage of the charge. The inclination of the voltage changes for the EDLC without and EDLC with the $10 \%$ CNT was almost the same. This result suggests that these EDLCs have almost the same electric capacitance. However, the voltage of the EDLC without the CNT addition reached $1.2 \mathrm{~V}$ in a shorter time than that of 


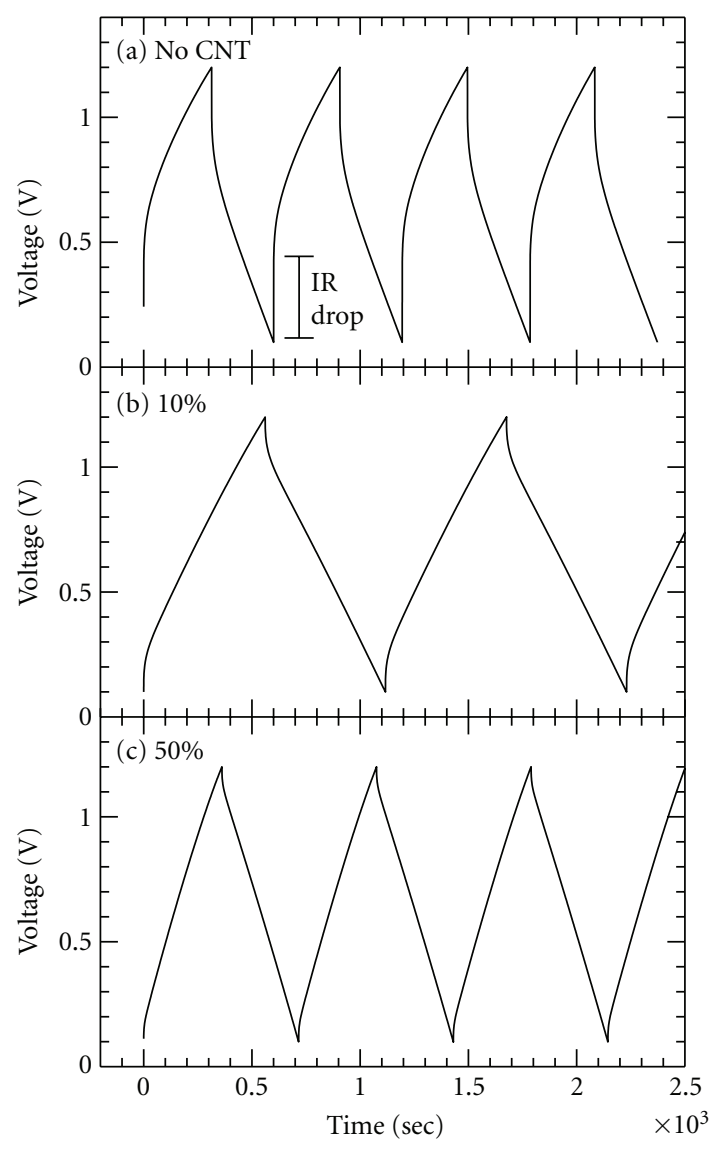

FIgURE 2: The charge-discharge curves of EDLCs fabricated with the following three types of polarizable electrodes: (a) those of exclusively activated carbon (no CNT) and those containing (b) 10 and (c) $50 \%$ CNTs.

the EDLC with the CNTs, because the IR drop is bigger. One cycle of the charge and the discharge on the EDLC without the CNT addition was $600 \mathrm{~s}$. On the other hand, that of the EDLC with the CNT addition was 1200 s. A longer cycle time indicates that a higher amount of electric energy is stored in the EDLC, because this measurement is carried out using a constant current.

The EDLC containing CNT at $50 \%$ was $0.02 \mathrm{~V}$ in the IR drop, which was lower than that of $10 \%$. However, one cycle of the charge and the discharge on this EDLC was as short as $700 \mathrm{~s}$, because the inclination of the voltage changes was higher than that of $10 \%$. Higher inclination suggests that the EDLC with the $50 \%$ CNT concentration is lower than that of $10 \%$ in the electrical capacitance.

An equivalent electric circuit model for the EDLCs, shown in Figure 3, was used to analyze the above chargedischarge curves. In this circuit, the equivalent capacitance $(C)$ is originated from the formation of the electrical double layer at the interface between surface of the polarizable electrodes and the electrolyte. Larger surface area of the polarizable electrode generally generates larger capacitance. The parallel resistance $\left(R_{p}\right)$ and the series resistance $\left(R_{s}\right)$ are connected to the capacitance. The parallel resistance

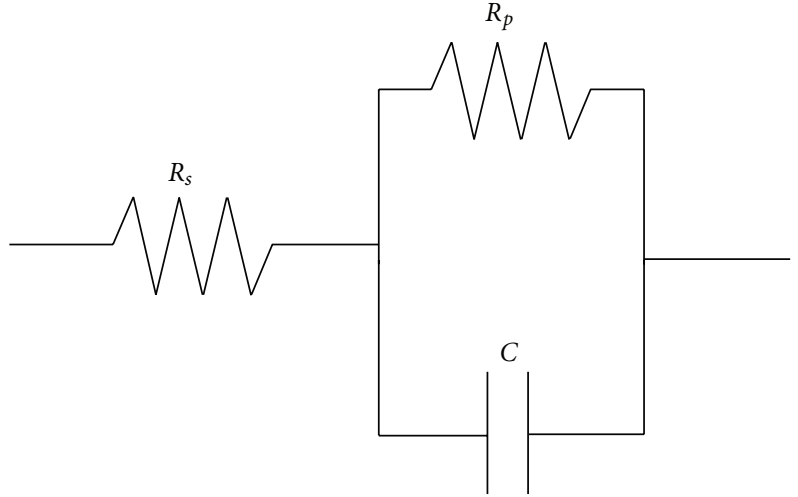

Figure 3: An equivalent electric circuit model for the EDLCs.

expresses the current leakage of the electrical double layer, which influences long-term energy storage. The series resistance consists of resistances of the polarizable electrodes and electrolyte, and contact resistance between the polarizable electrode and the collector electrode. The series resistance causes the IR drop and energy loss during the chargedischarge.

The equivalent series resistance $\left(R_{s}\right)$ of the EDLC was estimated from the value of the IR drop according to the following equation:

$$
R_{s}=\frac{V_{\mathrm{IR}}}{\left(I_{\text {charge }}-I_{\text {discharge }}\right)},
$$

where $V_{\text {IR }}$ is the voltage change at the time that the flowing current changes from the discharge current $I_{\text {discharge }}$ (negative value) to the charge current $I_{\text {charge }}$ (positive value). In this study, the value of $\left(I_{\text {charge }}-I_{\text {discharge }}\right)$ is $10 \mathrm{~mA}$. Therefore, the series resistance $\left(R_{s}\right)$ was estimated to be 45,4 , and $2 \Omega$ for the EDLCs without and those with CNT addition of $10 \%$ and $50 \%$, respectively. No dependence of the series resistance on the charge-discharge current was observed in the range between 0.5 and $50 \mathrm{~mA}$.

The inclination of the voltage change during the chargedischarge depends on the capacitance of the EDLC. Therefore, the capacitance $(C)$ of the EDLC was estimated using the mathematical fitting technique according to the following equation explaining the relationship between the charging time and the voltage:

$$
V(t)=R_{p} \cdot I_{\text {charge }}\left\{1-\exp \left(-\frac{t}{C \cdot R_{p}}\right)\right\}+V_{\mathrm{IR}},
$$

where $V(t)$ is the voltage as a function of the charging time $(t)$, the capacitance $(C)$, and the equivalent parallel resistance $\left(R_{p}\right)$.

The capacitance was estimated to be $2.4 \mathrm{~F}$ for both EDLC without and that with CNT at concentration of $10 \%$ from the above method. On the other hand, the EDLC with 50\% CNT was estimated to be as low as $1.2 \mathrm{~F}$, because the inclination of the voltage change was high and the charge-discharge cycle was short. No dependence of the capacitance on the charge current was observed in the range from 0.5 to $50 \mathrm{~mA}$. 
Figure 4(a) shows the dependence of the series resistance on the ratio of CNT to activated carbon in the polarizable electrode. Moreover, those of the EDLC containing the acetylene black, instead of the CNT, are also shown. In this figure, the ratio of 0 means the EDLC is fabricated exclusively with the activated carbon without using the CNT and acetylene black. The series resistance of the EDLC fabricated with exclusively of the activated carbon showed as high as value $45 \Omega$. When the CNTs were mixed with the activated carbon in the polarizable electrodes, the series resistance of the EDLC decreased with an increase in CNT concentration. The EDLC fabricated with a CNT concentration of $17 \%$ has a series resistance as low as $2 \Omega$. This series resistance was approximately one quarter of an EDLC fabricated at the same concentration of acetylene black. No series resistance changed with an increase in CNT concentration above $20 \%$. However, it increased to $2.5 \Omega$ in the face of the EDLC fabricated from $100 \%$ CNT, because clacks were formed in the polarizable electrodes by sinking them in the electrolyte.

The addition of the acetylene black to the polarizable electrode also decreased the series resistance of the EDLC. Both the EDLCs for concentration of the acetylene black at 50 and $100 \%$ are $4 \Omega$. No clacks were formed in the polarizable electrodes formed from exclusively acetylene black. However, the series resistance was higher than EDLCs with addition of the CNT.

Figure 4(b) shows the dependence of the specific capacitance $(\mathrm{F} / \mathrm{g})$ on ratio of the CNT or the acetylene black to the activated carbon in the polarizable electrode. In this figure, the value was indicated as the capacitance par the weight of polarizable electrode. For example, the EDLC fabricated from exclusively the activated carbon showed the $2.4 \mathrm{~F}$ in the capacitance. This EDLC is fabricated with two pieces of polarizable electrode containing carbon materials, such as activated carbon, CNT, or acetylene black (120 mg in total weight). Therefore, the specific capacitance of this EDLC was calculated to be $24 \mathrm{~F} / \mathrm{g}$.

No significant change in the capacitance was observed with mixture of the CNT and the acetylene black up to $17 \%$. The capacitance decreased with an increase in their ratio above 20\%. The EDLC fabricated exclusively from the CNT showed the capacitance of $4 \mathrm{~F} / \mathrm{g}$. This value is higher than that of the EDLC fabricated exclusively from acetylene black. However, these values are extremely lower than that of the EDLC fabricated from exclusively activated carbon.

The specific capacitances for the CNT and the activated carbon in the polarizable electrode were estimated by the mathematical fitting technique according to the following equation:

$$
C(x)=C_{\mathrm{CNT}} \frac{x}{100}+C_{\mathrm{AC}} \frac{(100-x)}{100},
$$

where $C(x)$ is specific capacitance for the fabricated polarizable electrode. $C_{\mathrm{CNT}}$ and $C_{\mathrm{AC}}$ are the specific capacitances for the CNT and the activated carbon, respectively. $x$ is ratio of the CNT to activated carbon. The result of the mathematical fitting is also shown in Figure 4(b).

Table 1 shows the specific capacitance for the CNT, the acetylene black, and the activated carbon estimated by the

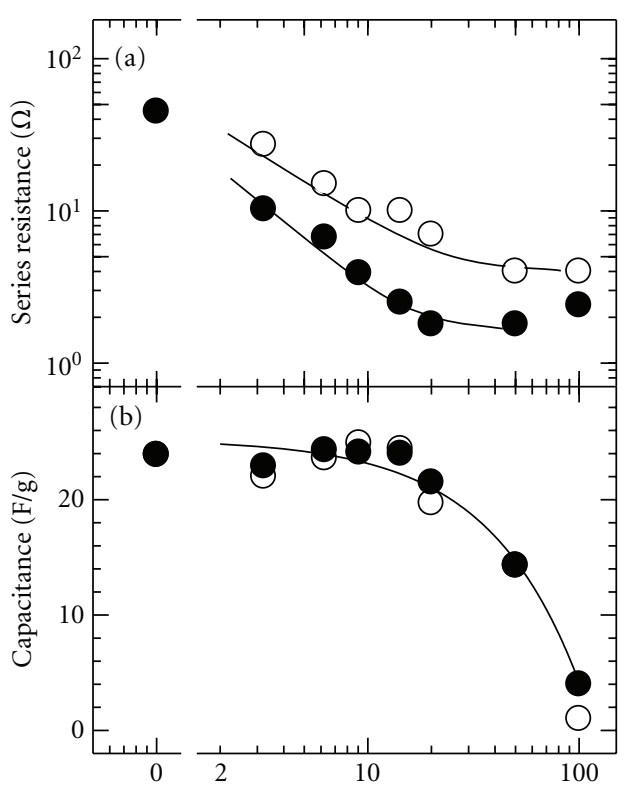

Ratio of CNT or acetylene black to activated carbon (\%)

$$
\mathrm{AB}
$$

FIGURE 4: (a) The dependence of (a) the series resistance and (b) the specific capacitance on the ratio of the CNT or the acetylene black to the activated carbon in the polarizable electrode.

TABLe 1: Specific capacitance of various materials used for the fabrication of EDLCs.

\begin{tabular}{lc}
\hline Material & $\begin{array}{c}\text { Specific capacitance } \\
(\mathrm{F} / \mathrm{g})\end{array}$ \\
\hline Carbon Nanotube & 4.5 \\
Acetylene black & 1.7 \\
Activated carbon & 24 \\
\hline
\end{tabular}

above method. The CNT and the acetylene black were 4.5 and $1.7 \mathrm{~F} / \mathrm{g}$ in specific capacitance, respectively. The CNT was higher than the acetylene black in the specific capacitance. However, they were lower than the activated carbon $(24 \mathrm{~F} / \mathrm{g})$.

Figure 5 shows the dependence of (a) the electrical energy and (b) the energy efficiency on ratio of the CNT or the acetylene black to the activated carbon in the polarizable electrode, whose weight is fixed at $60 \mathrm{mg}$. The electric energy $L$ stored in the EDLC was estimated by the discharge curve ranging between $1.2 \mathrm{~V}$ and $0.1 \mathrm{~V}$ with a constant current of $5 \mathrm{~mA}$ using the following equation:

$$
L=\int V(t) \cdot i d t .
$$

On the other hand, the energy efficiency $\eta$ was defined by the ratio between the discharged and the charged electric power using the following equation:

$$
\eta=\frac{L_{\text {discharge }}}{L_{\text {charge }}} \times 100,
$$




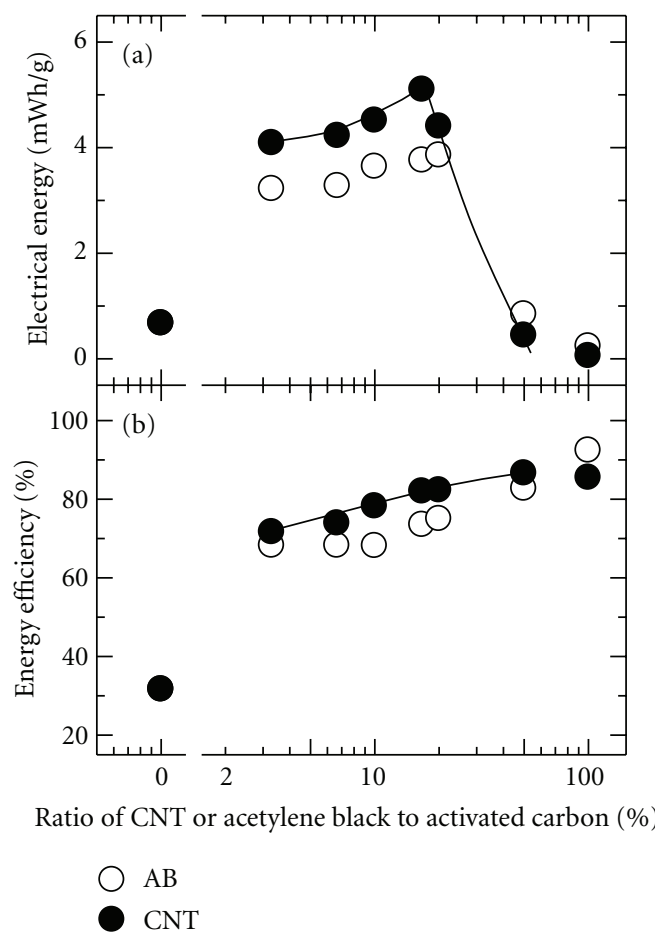

Figure 5: The dependence of (a) the electrical energy and (b) the energy efficiency on ratio of the CNT or the acetylene black to the activated carbon in the polarizable electrode.

where $L_{\text {charge }}$ and $L_{\text {discharge }}$ are, respectively, the electric power charged and discharged between the voltage of $0.1 \mathrm{~V}$ and $1.2 \mathrm{~V}$ with a constant current of $5 \mathrm{~mA}$.

The EDLC fabricated using exclusively activated carbon without the addition of any conducting materials showed a low electric energy of $0.7 \mathrm{mWh} / \mathrm{g}$. When the CNTs were added to the polarizable electrodes up to $15 \%$, the electric energy increased up to $5.1 \mathrm{mWh} / \mathrm{g}$ according to the increase in CNT concentration. The electric energy was on the contrary decreased by increasing the CNT concentration above $20 \%$. The EDLCs with addition of the acetylene black also have the same trend in the electrical energy. However, the electric energy for EDLCs fabricated with CNTs was higher than that of those fabricated with acetylene black.

A low energy efficiency of $31 \%$ was observed for the EDLC fabricated exclusively from activated carbon as shown in Figure 5(b). The energy efficiency was improved by the addition of CNTs to the polarizable electrodes and linearly increased according to the increase in the CNT concentration. A high energy efficiency of $86 \%$ was observed for the EDLC with a $50 \%$ CNT concentration. The energy efficiency of the EDLCs with addition of the CNT was higher than that of the acetylene black.

Figure 6 shows the dependence of (a) the capacitance and (b) the series resistance on the weight and the thickness of the polarizable electrode in EDLC. The thickness of the polarizable electrode is increased by an increase in its weight, since the polarizable electrode is fixed at $11.5 \mathrm{~mm}$ in the diameter. The CNT or the acetylene black in the

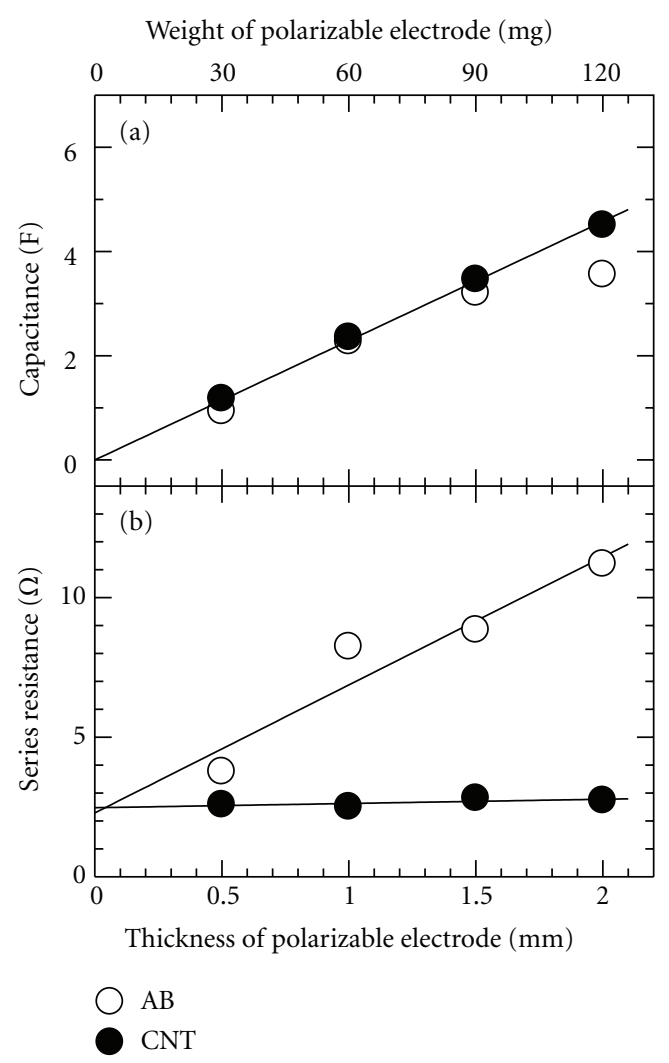

FIGURE 6: The dependence of (a) the capacitance and (b) the series resistance on the weight and the thickness of the polarizable electrode.

polarizable electrodes was fixed at $10 \%$ in the weight ratio. Both of the EDLCs fabricated with the CNT or the acetylene black linearly increased from 1.5 to $4.5 \mathrm{~F}$ in capacitance with an increase in the thickness of polarizable electrodes. The specific capacitances at various thicknesses were almost of the same value of $24 \mathrm{~F} / \mathrm{g}$. The series resistance of the EDLCs added with the acetylene black was increased from 3.7 to $11 \Omega$ with increasing the thickness of polarizable electrode. On the other hand, the series resistance of the EDLCs fabricated with the CNT slightly increased from $2.5 \Omega$ to $2.8 \Omega$ with an increase in the thickness of polarizable electrode. The series resistance at the thickness of $0 \mathrm{~mm}$ was estimated to be $2.5 \Omega$ by extrapolation method. The EDLC formed with the acetylene black also showed the same value. These series resistances represent the contact resistance between the polarizable electrode and the counter electrode, because the resistances of the polarizable electrode and the electrolyte are neglected at the thickness of $0 \mathrm{~mm}$. Therefore, these results indicate that the series resistance of the EDLCs fabricated using the CNT are governed by the contact resistance, since the resistance of the polarizable electrode containing CNT is low enough.

Figure 7 shows the schematic model comparing the electrical conduction in the polarizable electrodes with addition of (a) the acetylene black or (b) the CNT. Acetylene black is particle with a diameter of $50-60 \mathrm{~nm}$, which is smaller than activated carbon by a one-30th to a one-60th approximately. 


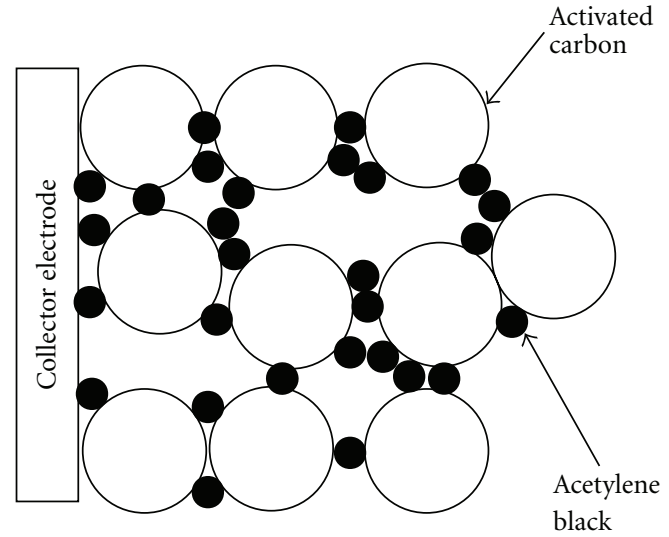

(a) Acetylene black

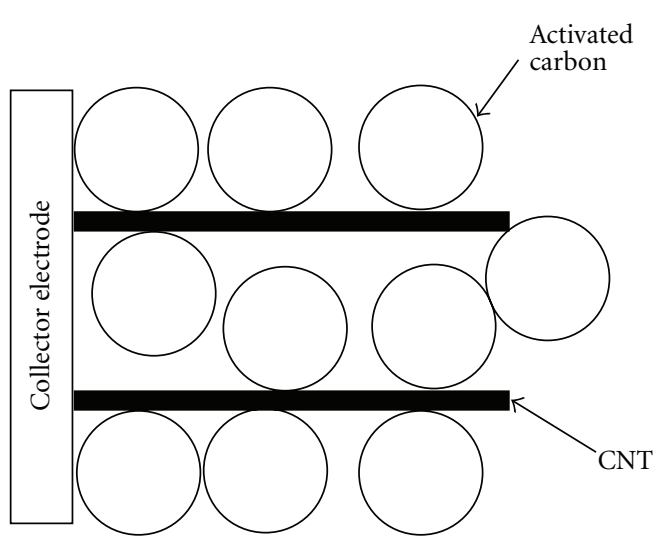

(b) Carbon Nanotube (CNT)

FIGURE 7: The schematic model comparing the electrical conduction in the polarizable electrodes with addition of (a) the acetylene black and (b) the CNT.

The particles of the acetylene black cover activated carbon particles in the polarizable electrode. The charge and the discharge currents flow through a large number of the acetylene black particles on the activated carbon. In this conduction mechanism, the acetylene black particles must connect to each other in order to effectively decrease the resistance of polarizable electrode. Although CNT has cylindrical shape with the diameter of $10 \mathrm{~nm}$ which is size a similar to that of acetylene black the length of $\mathrm{CNT}$ is as long as $100 \mu \mathrm{m}$. Therefore, one piece of CNT connects large number of activated carbon particles, because the length of CNT is longer than activated carbon by approximately 30 50 times. Therefore, CNT forms the electrical conduction mechanism in the polarizable electrode, which has higher conductivity comparing with acetylene black.

Activated carbon is a particle with the specific surface area of $2000 \mathrm{~m}^{2} / \mathrm{g}$. Therefore, it is widely used as a constitution of the EDLC. In this study, the specific capacitance for the activated carbon was $24 \mathrm{~F} / \mathrm{g}$. On the other hand, CNT also seems to have large surface area, because it has cylindrical shape with small diameter. The small diameter expects that CNT is more suitable constitution of the EDLC than activated carbon. However, the CNT showed $4.5 \mathrm{~F} / \mathrm{g}$ in specific capacitance, which was smaller than the activated carbon. Small capacitance in this study suggests that only small surface area of CNT contributes to the double-layer capacitor. One possibility of this reason is the hydrophobic surface of CNT that causes its surface to be hardly wet with electrolyte.

EDLC fabricated from exclusively activated carbon shows high capacitance of 2.4 F. However, the electric energy stored in its EDLC is as low as $0.7 \mathrm{mWh} / \mathrm{g}$, because its series resistance has value as high as $45 \Omega$. High series resistance loses electric energy during the charge and the discharge and also decreases the energy efficiency. The CNT addition into the polarizable electrode decreases the series resistance of the EDLC up to $2 \Omega$. Moreover, the EDLC with CNT addition increases to $5.1 \mathrm{mWh} / \mathrm{g}$ in the electric energy, because lower series resistance causes lower IR drop and longer charging time. The small series resistance prevents losing electric energy during the charge and discharge. Therefore, the higher electric efficiency is observed from the EDLC with the addition of CNT. The addition of acetylene black also has the same trend in these characteristics. However, CNT is more effective than acetylene black as a conducting material.

The significant decrease in the capacitance of the EDLC with the addition of CNT was observed above $20 \%$, because the specific capacitance of CNT is lower than that of activated carbon. The decrease of the capacitance leads to the lower stored electric energy. However, the efficiency increases with an increase in the CNT concentration, since the series resistance is decreased.

The CNT addition decreases the series resistance of EDLCs, because of its high aspect ratio. However, the CNT addition above $20 \%$ does not decrease the series resistance, because the series resistance becomes governed by the contact resistance between the polarizable electrode and the counter electrode. On the other hand, the capacitance decreases with an increase in the CNT concentration above $20 \%$, because of low specific capacitance of CNT. These results suggest that the $\mathrm{CNT}$ is suitable material as the conducting material under the concentration of $20 \%$.

\section{Summary}

The CNT addition into the polarizable electrodes up to $20 \%$ reduces the series resistance of the EDLC from 45 to $2 \Omega$. The reduction of the series resistance increases the electrical energy stored in the EDLC, and also prevents losing the energy loss during the charge and the discharge. However, the CNT addition does not increase the capacitance, because most of surface area of the CNT does not contribute to the electrical double layer capacitor. The EDLC with the $20 \%$ CNT addition shows the capacitance of $24 \mathrm{~F} / \mathrm{g}$, which is mainly contributed by activated carbon.

The EDLC with the CNT addition has lower than that of acetylene black in the series resistance. Since the series 
resistance of the EDLC was decreased by the CNT addition, the EDLC achieves to increase the electric energy stored in it. Moreover, the energy efficiency for the charge and the discharge is also improved.

\section{Acknowledgments}

The author would like to thank Mr. Y. Miyamoto of the Technical Service Coordination Office, Tokai University, for the SEM investigations and Mrs. R. Kuwabara, H. Ino, M. Imano, H. Yoshida, Y. Totani, K. Koizumi, and K. Imaizumi for useful discussions.

\section{References}

[1] H. I. Becker, "Low voltage electrolytic capacitor," U.S. Patent $2800616,1957$.

[2] A. Nishino, "Capacitors: operating principles, current market and technical trends," Journal of Power Sources, vol. 60, no. 2, pp. 137-147, 1996.

[3] K. Naoi and K. Machida, "Electrochemical Capacitors and Automobiles," Kinzoku, vol. 74, p. 1299, 2004.

[4] M. Endo, T. Maeda, T. Takeda et al., "Capacitance and poresize distribution in aqueous and nonaqueous electrolytes using various activated carbon electrodes," Journal of The Electrochemical Society, vol. 148, no. 8, pp. A910-A914, 2001.

[5] T. Morimoto, K. Hiratsuka, Y. Sanada, and K. Kurihara, "Electric double-layer capacitor using organic electrolyte," Journal of Power Sources, vol. 60, no. 2, pp. 239-247, 1996.

[6] T. W. Ebbesen, H. J. Lezec, H. Hiura, J. W. Bennett, H. F. Ghaemi, and T. Thio, "Electrical conductivity of individual carbon nanotubes," Nature, vol. 382, pp. 54-56, 1996.

[7] E. Frackowiak, K. Metenier, V. Bertagna, and F. Beguin, "Supercapacitor electrodes from multiwalled carbon nanotube," Applied Physics Letters, vol. 77, no. 15, artcle 2421, 3 pages, 2000.

[8] E. Frackowiak, K. Jurewicz, S. Delpeux, and F. Beguin, "Nanotubular materials for supercapacitors," Journal of Power Sources, vol. 97-98, pp. 822-825, 2001.

[9] K. H. An, K. K. Jeon, W. S. Kim et al., "Characterization of supercapacitors using singlewalled carbon nanotube electrodes," Journal of the Korean Physical Society, vol. 39, pp. S511-S517, 2001.

[10] B. J. Yoon, S. H. Jeong, K. H. Lee, H. S. Kim, C. G. Park, and J. H. Han, "Electrical properties of electrical double layer capacitors with integrated carbon nanotube electrodes," Chemical Physics Letters, vol. 388, pp. 170-174, 2004.

[11] C. Emmenegger, P. Mauron, P. Sudan et al., "nvestigation of electrochemical double-layer (ECDL) capacitors electrodes based on carbon nanotubes and activated carbon materials," Journal of Power Sources, vol. 124, no. 1, pp. 321-329, 2003.

[12] C. Portet, P. L. Taberna, P. Simon, and E. Flahaut, "Influence of carbon nanotubes addition on carbon-carbon supercapacitor performances in organic electrolyte," Journal of Power Sources, vol. 139, no. 1-2, pp. 371-378, 2005.

[13] C.-G. Liu, H.-T. Fang, D.-W. Wang, F. Li, M. Liu, and H.M Cheng, "Electrochemical Capacitance Characteristics of Activated Carbon Electrode Material with a Multi-Walled Carbon Nanotube Additive," New Carbon Materials, vol. 20, no. 3, pp. 205-210, 2005.

[14] Y. Show and K. Imaizumi, "Decrease in equivalent series resistance of electric double-layer capacitor by addition of carbon nanotube into the activated carbon electrode," Diamond and Related Materials, vol. 15, no. 11-12, pp. 2086-2089, 2006.

[15] Y. Show and K. Imaizumi, "Electric double layer capacitor with low series resistance fabricated by carbon nanotube addition," Diamond and Related Materials, vol. 16, no. 4-7, pp. 11541158, 2007. 

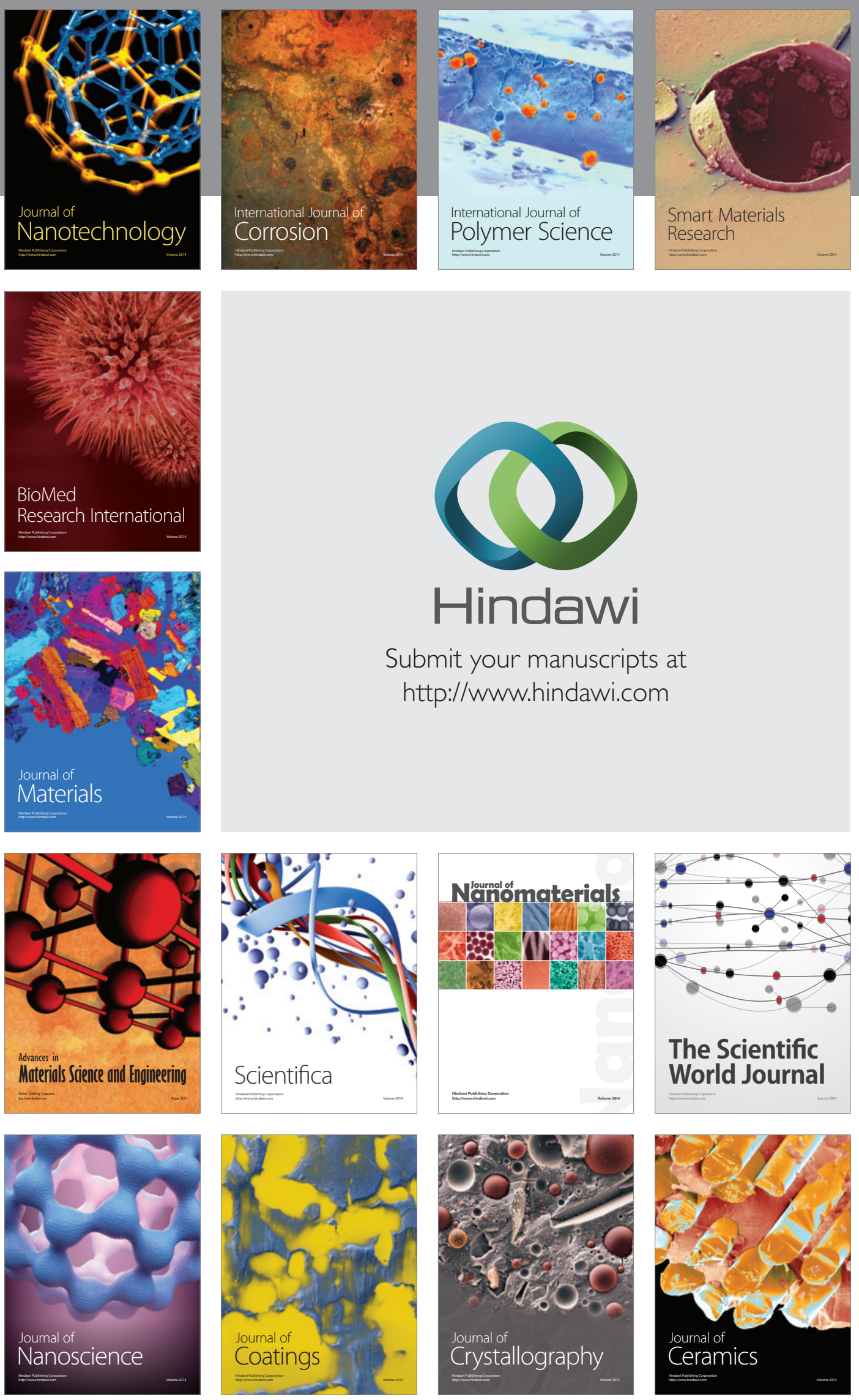

The Scientific World Journal

Submit your manuscripts at

http://www.hindawi.com

\section{World Journal}

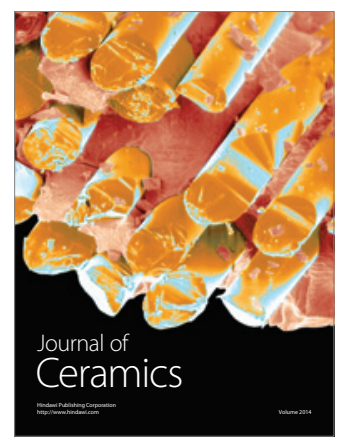

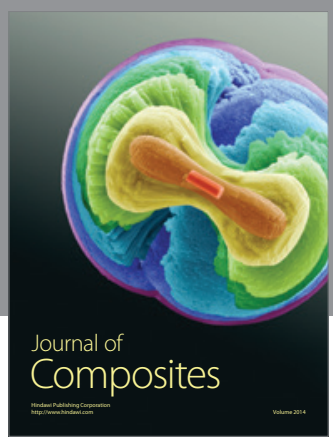
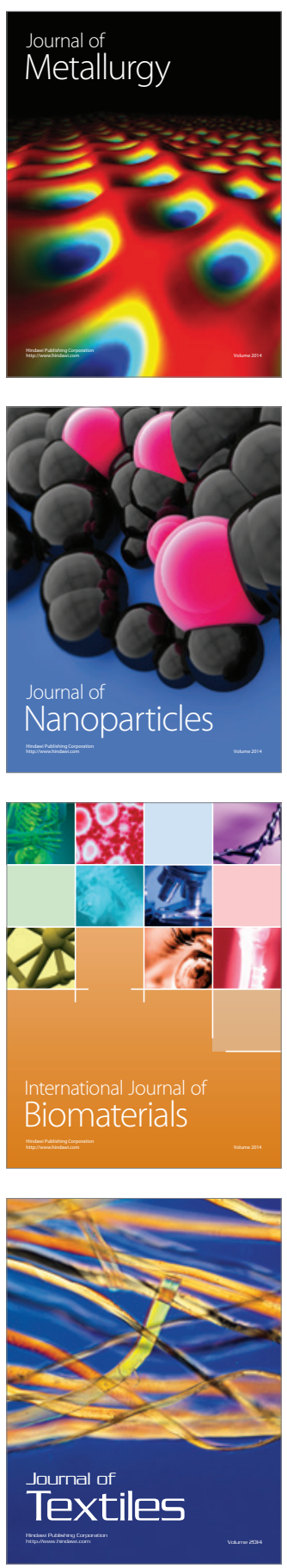